\title{
Molecular Dynamics Simulations Reveal Specific Interactions of Post- translational Palmitoyl Modifications with Rhodopsin in Membranes
}

\author{
Bjoern E. S. Olausson, ${ }^{\dagger}{ }^{\ddagger}$ Alan Grossfield, ${ }^{\S}$ Michael C. Pitman, ${ }^{\perp}$ Michael F. Brown, ${ }^{\|, \#}$ Scott E. Feller, ${ }^{\otimes}$ \\ and Alexander Vogel ${ }^{*}$ \\ ${ }^{\dagger}$ Institute of Pharmacy, Martin-Luther-University Halle-Wittenberg, D-06120 Halle/Saale, Germany \\ ${ }^{\ddagger}$ Institute for Medical Physics and Biophysics, University of Leipzig, D-04107 Leipzig, Germany \\ ${ }^{\S}$ Department of Biochemistry and Biophysics, University of Rochester Medical Center, Rochester, New York 14642, United States \\ ${ }^{\perp}$ IBM T.J. Watson Research Center, Yorktown Heights, New York 10598, United States \\ "Department of Chemistry and Biochemistry and "Department of Physics, University of Arizona, Tucson, Arizona 85721, United \\ States \\ ${ }^{\otimes}$ Department of Chemistry, Wabash College, Crawfordsville, Indiana 47933, United States
}

Supporting Information

ABSTRACT: We present a detailed analysis of the behavior of the highly flexible posttranslational lipid modifications of rhodopsin from multiple-microsecond all-atom molecular dynamics simulations. Rhodopsin was studied in a realistic membrane environment that includes cholesterol, as well as saturated and polyunsaturated lipids with phosphocholine and phosphoethanolamine headgroups. The simulation reveals striking differences between the palmitoylations at Cys322 and Cys323 as well as between the palmitoyl chains and the neighboring lipids. Notably the palmitoyl group at Cys 322 shows considerably greater contact with helix $\mathrm{H} 1$ of rhodopsin, yielding frequent chain upturns with longer reorientational correlation times, and relatively low order parameters. While the palmitoylation at Cys323 makes fewer protein contacts and has increased order

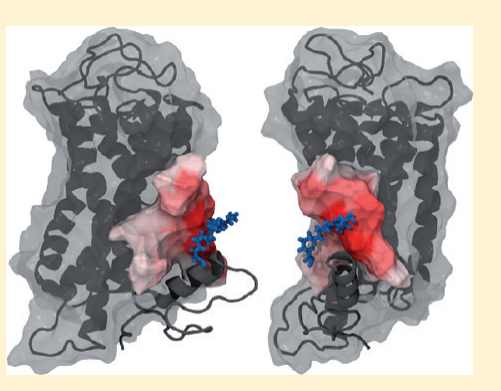
compared to Cys322, it nevertheless exhibits greater flexibility with smaller order parameters than the stearoyl chains of the surrounding lipids. The dynamical structure of the palmitoylations-as well as their extensive fluctuations-suggests a complex function for the post-translational modifications in rhodopsin and potentially other G protein-coupled receptors, going beyond their role as membrane anchoring elements. Rather, we propose that the palmitoylation at Cys323 has a potential role as a lipid anchor, whereas the palmitoyl-protein interaction observed for Cys322 suggests a more specific interaction that affects the stability of the dark state of rhodopsin.

\section{INTRODUCTION}

Rhodopsin is an integral membrane protein responsible for the detection of light in the visual system of vertebrates and invertebrates. It belongs to the large $G$ protein-coupled receptor (GPCR) superfamily, which plays a crucial role in many biological signaling processes. ${ }^{1}$ Notably, $\sim 50 \%$ of all current drugs target these proteins. ${ }^{2}$ For many years rhodopsin has served as the prototypical GPCR because it was the first member for which an atomic structure was determined; ${ }^{3}$ consequently it remains the best understood GPCR from a biophysical perspective. The conformational changes of rhodopsin induced by light absorption have been studied extensively, and structural information is available for several of its photoproducts ${ }^{4-7}$ Yet, the molecular basis of its activation remains under extensive discussion at present, ${ }^{4,5,8}$ so that a clear consensus picture of the reaction mechanism has not emerged.

One of the important features revealed by the crystal structure of rhodopsin-besides the canonical GPCR structural motif of seven transmembrane helices-was an additional eighth helix that is roughly parallel to the membrane surface. ${ }^{3}$
Its amino acids are highly conserved across class A GPCRs and it is now believed this feature is a common structural element among them. ${ }^{9}$ This proposal is supported by the observation that almost all known high-resolution crystal structures of class A GPCRs exhibit this helix. ${ }^{3,10-14}$ Other investigations revealed that helix $\mathrm{H} 8$ is involved in the binding, and possibly the activation, of the G protein. ${ }^{9,15,16}$ Specifically helix H8 is in direct contact with the $\alpha$ and $\gamma$ subunits of the associated G protein (transducin) and controls their affinity for rhodopsin. ${ }^{17-19}$ In many GPCRs, a further remarkable aspect is that one or more cysteines close to helix $\mathrm{H} 8$ are post-translationally modified by palmitic acid. These palmitoylations-or more correctly thio $(S)$-acylations-are a common modification of membrane proteins having very different cellular location, topology, and function. In some instances, the palmitoyl chains are responsible for anchoring peripheral membrane proteins to the membrane surface, while in other cases they are attached to

Received: November 17, 2011

Published: January 23, 2012 
transmembrane proteins that are already stably membranebound. $^{20}$

For GPCRs such as rhodopsin, protease-activated receptor type $1, \beta_{2}$-adrenergic receptor, human prostacyclin receptor, thyrotropin-releasing hormone receptor, human prostanoid thromboxane $\mathrm{A}_{2}$ receptor, and CCR5 receptor the palmitoylation sites are found in the conserved region following helix H7. Evidence has recently been gathered indicating that palmitoylation (sometimes together with isoprenylation) modulates G protein coupling., ${ }^{91-23}$ Removing the palmitoylation leads to severe effects on function; ${ }^{9,21-27}$ nevertheless, in most cases the receptor is still able to activate $G$ proteins. Often, however, removal leads to a lack of functionality in distal regions, such as those implicated in phosphorylation due to allosteric effects. For instance, it has been reported that (de)palmitoylation of the human prostacyclin receptor occurs upon agonist binding, which therefore may provide a switch mechanism for structural changes in the proximity of the palmitoylated cysteines. ${ }^{24}$ Additionally, the ability of the thyrotropin-releasing hormone receptor to become phosphorylated is severely inhibited by mutation of the palmitoylated cysteines. $^{25}$ Similar behavior has been shown for opsin (which is the protein moiety of rhodopsin). ${ }^{27}$ For the human thromboxane A2 receptor, palmitoylation not only mediates efficient coupling to the $G$ protein, but is also needed for internalization of the receptor, and even affects which pathway is followed. ${ }^{23}$ Other roles for palmitoylation include determining the subcellular location of the GPCR and its diffusion properties, ${ }^{26}$ which is also supported by theoretical studies of the effects of protein palmitoylation. ${ }^{28}$

In rhodopsin, the two cysteines in positions 322 and 323 at the C-terminal end of helix $\mathrm{H} 8$ are palmitoylated, ${ }^{29}$ and it is assumed they contribute to stabilizing helix $\mathrm{H} 8$ at the lipidwater interface of the membrane. However, the exact function of the palmitoylations is still unclear. Their removal does not seem to have a large effect on the structure of rhodopsin and the interaction with the ligand, ${ }^{27,30}$ although activation of the $G$ protein transducin is slightly increased and the C-terminus of rhodopsin stabilized by their presence. ${ }^{30}$ A potential role for palmitoylation involves acting as a mediator of lipid-protein interactions, or more generally to perturb the material properties of the membrane. It is well established that rhodopsin reacts very sensitively to its lipid environment. ${ }^{31}$ Material properties such as curvature stress of the surrounding membrane can considerably shift the equilibrium between the two intermediates metarhodopsin I (MI) and metarhodopsin II (MII) in the activation process. ${ }^{32}$ For example, replacement of palmitic acid at the $s n-1$ position of the lipids by oleic acid shifts the equilibrium toward the activated MII state. ${ }^{33,34}$ Moreover, it has recently been concluded that specific interactions between rhodopsin and the head groups and acyl chains of the lipids in the first surrounding layer are important for the MI-MII equilibrium. ${ }^{33}$ Modeling studies have supported the observations of specific lipid-protein interactions, e.g., a preference for solvation by polyunsaturated lipid acyl chains. ${ }^{35,36}$ A region comprised of helix $\mathrm{H} 8$ and surrounding amino acids clearly favors polyunsaturated over saturated acyl chains. Notably the lipid composition of the retinal disk membrane contains a high proportion of phospholipids with highly polyunsaturated acyl chains $^{31}$ that have been studied extensively. ${ }^{37-40}$

Despite rather comprehensive knowledge of the effects of palmitoylation on the function of GPCRs, very little is known about the structure and dynamics of the acyl chains themselves.
Studies of rhodopsin with fluorescently labeled palmitoylations have shown they are well embedded in the membrane, and that the terminal methyl groups are located deep in the hydrophobic core. ${ }^{41}$ They are often resolved in the recent crystal structures, but exhibit very different conformations. ${ }^{42-51}$ However, it is unlikely they exhibit any preferred conformation in the more dynamic membrane environment. Studies of palmitoylations of peripheral membrane proteins show them to be more flexible than lipid acyl chains, and therefore much more flexible than the rather rigid protein backbone structures. ${ }^{52-54}$ To directly investigate the palmitoyl chains in rhodopsin, we carried out molecular dynamics (MD) simulations of a single protein in a hydrated lipid bilayer membrane. The bilayer comprised a 2:2:1 mixture of 1-stearoyl-2- docosahexaenoyl-sn-glycero-3-phosphatidylethanolamine (SDPE), 1-stearoyl-2-docosahexaenoyl-snglycero-3-phosphatidylcholine (SDPC), and cholesterol. Recent advances have opened the door to microsecond-time scale simulations of membrane proteins in full atomic detail, thus providing important molecular level insight into their structure, dynamics, and function. ${ }^{35,55-57} \mathrm{We}$ analyzed a data set composed of 26 simulations, each $\sim 100 \mathrm{~ns}$, where the bilayers were constructed independently, as well as an additional single $\mathrm{MD}$ trajectory of $1.6 \mu \mathrm{s}$ length. Extensive sampling in a realistic membrane environment is particularly important for the study of the rhodopsin palmitoylations, whose structure and dynamics are potentially coupled both to conformational substates of the protein ${ }^{8}$ and to fluctuations in the lipid environment. Our results indicate that the two palmitoylations behave very differently despite their close location in two neighboring cysteines, suggesting unique roles for these posttranslational modifications.

\section{METHODS}

The 1.6- $\mu$ s MD simulation involved a rhodopsin molecule (PDB code $1 \mathrm{U} 19^{58,59}$ ) embedded in a lipid bilayer of 49 SDPC, 50 SDPE, and 24 cholesterol (Chol) molecules hydrated by 7400 TIP3 waters, $14 \mathrm{Na}^{+}$, and $16 \mathrm{Cl}^{-}$ions, giving a total of 43222 atoms. A snapshot from the simulation is shown in Figure 1, which depicts the composition of the simulation cell. Details of the system setup and equilibration have been reported elsewhere. ${ }^{35}$ Briefly, simulations were performed in the dark state at $311 \mathrm{~K}$ in the NVE ensemble using the Program BlueMatter. Periodic boundary conditions were employed with dimensions of 56.5 $\times 79.2 \times 95.5 \AA$. The membrane area was chosen based on NMR measurements of the stearoyl chain order parameters and subsequently compared to these experimental data. ${ }^{60}$ The CHARMM27 force field was used for the protein, ${ }^{61}$ and CHARMM parameters for the saturated chain, ${ }^{62}$ polyunsaturated chain, ${ }^{39}$ and cholesterol ${ }^{63}$ were utilized for the membrane lipid components. Construction and equilibration were performed in CHARMM version 27 as detailed elsewhere. ${ }^{35}$ Long-range electrostatic interactions were calculated using the particle-particle-particle mesh Ewald summation technique, ${ }^{64,65}$ with a $128^{3}$ grid for the fast Fourier transform, a chargeinterpolation distance of four mesh points, and the Ewald $\alpha$ value set to $0.35 \AA^{-1}$. Real-space electrostatics and repulsion-dispersion were smoothly truncated at $10 \AA$. All bonds containing hydrogen were constrained to their equilibrium values using the RATTLE algorithm, ${ }^{66}$ allowing us to run dynamics with a 2 -fs time step using the velocity-Verlet integrator. The 26 simulations, each of length $\sim 100 \mathrm{~ns}$, were performed identically with a unique set of lipid conformations and locations used to generate initial conditions for each simulation. Full details are found in ref 35 .

\section{RESULTS}

Our results are based on $\sim 4.3 \mu \mathrm{s}$ of $\mathrm{MD}$ simulation (comprising one trajectory of $\sim 1.6 \mu$ s length and 26 different 


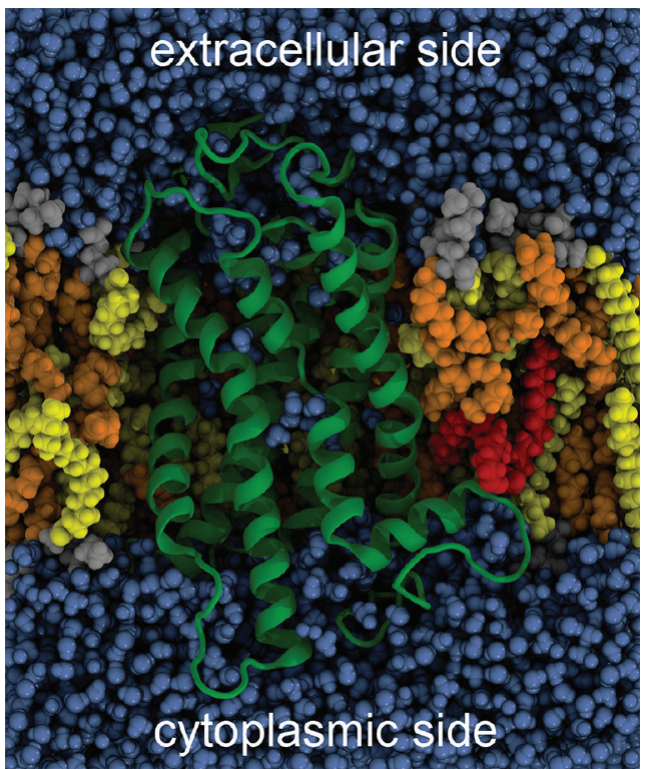

Figure 1. Snapshot of the MD simulation cell showing the composition of the system. The polypeptide backbone of rhodopsin is depicted in green with both lipid modifications shown in a van der Waals (vdW) representation in red. The lipid vdW surfaces are indicated with the head groups and glycerol backbone in gray, the DHA chains in orange, and the STEA chains in yellow. The extracellular side of the membrane is up and the cytoplasmic side is down. Note that one of the palmitoylations is in contact with the helix bundle and exhibits a kink leading to a chain upturn. The figure was prepared using VMD. ${ }^{67}$

100-ns simulations, all with individual starting configurations). They provide an unprecedented level of detail on the structure and fluctuations of the rhodopsin palmitoylations in a membrane environment. The most striking observation was that the lipid modifications make frequent contacts with the transmembrane helices. Therefore, we evaluated the position of the palmitoyl chain of Cys 322 relative to the protein using a contact-map representation, as shown in Figure 2A,B. The distances between all heavy atoms of the palmitoyl chain and all heavy atoms of the amino acids were calculated, where each distance less than $4 \AA$ was defined as a contact. The sum of all contacts was accumulated over all simulations, and this index was mapped onto the surface representation of the protein, where the more intensely colored regions indicate more frequent contacts. For Cys322, the vast majority of contacts occurred on helix $\mathrm{H} 1$ in the region between residues 45-62. Among residues on the protein, the greatest overall number of contacts was recorded for Pro53 in helix H1. Fewer encounters with residues in helices other than $\mathrm{H} 1$ were also observed, with helix $\mathrm{H} 7$ receiving $\sim 5 \%$ as many as helix $\mathrm{H} 1$; but no other region of the protein made reproducible contacts with the Cys322 palmitoyl residue. The overall contacts for Cys 323 (Figure 2C,D) are noticeably lower than shown for the palmitoylation on Cys322. Again, most contacts were made with $\mathrm{H} 1$ between residues 48-61; residue Pro53 made contact most frequently. Figure 2 also includes snapshots of palmitoyl configurations that illustrate typical states for these chains. Neither chain is observed in a fully extended conformation that places palmitoyl methyl groups at the center of the bilayer. Rather these modifications are typically found in more highly kinked states, with frequent chain upturns associated with close contacts to the protein.
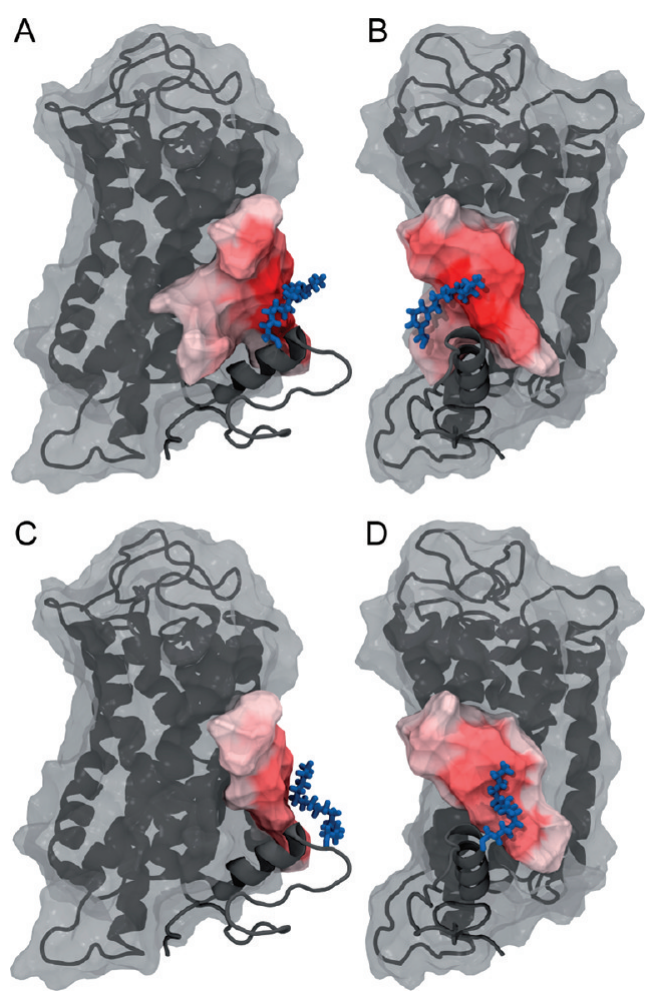

Figure 2. Depiction of the amino acids which experience close contacts with the lipid modification attached to Cys322 (panels A and B) and to Cys323 (panels C and D) shown from two different angles. The membrane extracellular side is up and the cytoplasmic side is down. Rhodopsin is shown with a semitransparent surface. All amino acids that experience contacts are shown in a nontransparent surface representation. The intensity of the red color indicates the number of contacts with the lipid modification. The palmitoylation at Cys 322 shows many contacts with helix $\mathrm{H} 1$ and some with helix $\mathrm{H} 7$. The palmitoylation at Cys323 shows a similar pattern, but with a narrower distribution and fewer contacts overall. The figure was prepared using VMD. ${ }^{67}$

To quantify contacts between the palmitoylations and the phospholipid acyl chains in the bilayer, we computed the radial distribution function, RDF or $g(r)$, as displayed in Figure 3. Because the properties of the saturated stearic acid (STEA) and polyunsaturated docosahexaenoic acid (DHA) groups differ significantly, we evaluated the distances between each of the phospholipid chains and the palmitoyl groups using the $g(r)$ function implemented in VMD. ${ }^{67}$ From Figure 3 we see that DHA chains are more likely to surround the palmitoylations than STEA chains, a result that parallels earlier findings that rhodopsin is preferentially solvated by polyunsaturated fatty acid (PUFA) chains. ${ }^{35,36}$ This finding suggests the palmitoylations do not optimize their interactions with phospholipids, which would be increased by close packing with saturated chains that maximize van der Waals (vdW) attractions. To investigate the possibility of preferential interactions with lipids that exhibit a phosphatidylcholine (PC) or phosphatidylethanolamine (PE) headgroup, we repeated the radial distribution function calculation separately (Supporting Information, SI). However, we found no evidence for selectivity of the palmitoylations for lipids with certain head groups.

The disordered nature of the palmitoyl chains arises from geometric restraints imposed by interactions with specific residues on the protein (Figure 2), and from the chaotic tendencies of the PUFA chains in the lipid matrix. Their 


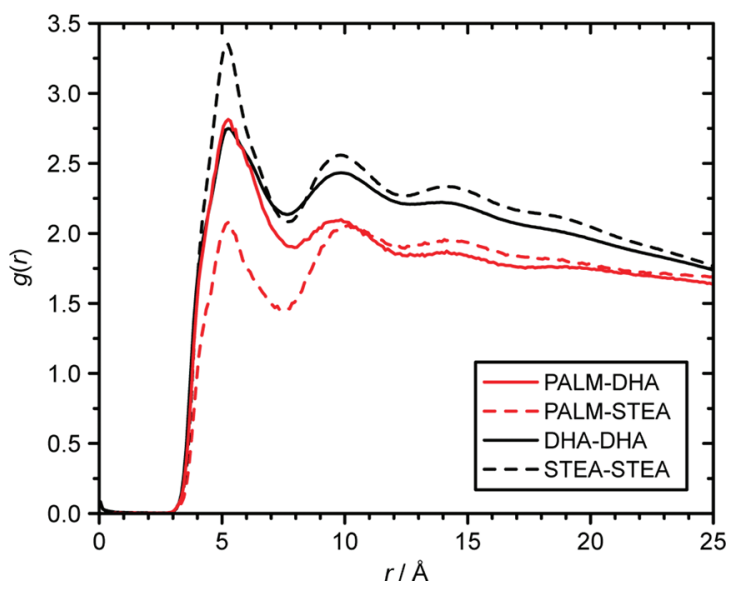

Figure 3. Radial pair distribution functions $g(r)$ of both lipid modifications of rhodopsin with the individual DHA (red, solid line) and STEA (red, dashed line) chains. For comparison the RDFs for DHA chains with themselves (black, solid line) and STEA chains with themselves (black, dashed line) are also shown. When analyzing the direct neighbors of the palmitoylations (red lines) at small distances $r$ significant differences are observed, indicating they prefer DHA over STEA chains. Note that DHA (solid lines) is about as likely to have a palmitoyl chain (PALM) as its neighbor as another DHA chain. In contrast STEA (dashed lines) has many more STEA chains close to it than palmitoyl chains.

mobility can be quantified by computing a segmental order parameter as a function of position along the palmitoyl chain, which includes both the geometric effects as well as the amplitudes of the orientational fluctuations of the $\mathrm{CH}_{2}$ groups. We have chosen the deuterium order parameter, $S_{\mathrm{CD}}$, because it can be related to the quadrupolar splitting measured directly in a solid-state ${ }^{2} \mathrm{H}$ NMR experiment. ${ }^{37,38}$ It is thus a testable prediction of our simulations. The order parameters were calculated for both palmitoylations individually and compared to the STEA chains. The formula $S_{\mathrm{CD}}={ }^{1} /{ }_{2}\left\langle 3 \cos ^{2} \theta-1\right\rangle$ was used, where $\theta$ is the orientation of the $\mathrm{C}-\mathrm{D}$ ( or $\mathrm{C}-\mathrm{H}$ ) bond vector with respect to the membrane normal, and the angular brackets denote an ensemble or time average. Notably the simulated order parameters (Figure 4) for both lipid modifications are significantly decreased versus the stearoyl chains of the lipids, indicating large amplitude motions or more disordered conformations. Additionally, a large difference is observed between the two lipid modifications, with the order parameters for Cys322 noticeably lower than those for Cys323. The Cys322 chain, which makes more frequent protein contacts, tends to adopt specific conformations dictated by interactions with rhodopsin, and it is more likely to be highly kinked. It also exhibits slower dynamics, with reorientational correlation functions that have longer relaxation times than the Cys323 palmitoylation (SI).

In saturated acyl chains, the order parameters can be related to the projection of the chain length along the membrane normal (taken here as the $z$-axis); e.g., higher magnitude order parameters are associated with greater alignment along $z$, and therefore longer chains on average. ${ }^{60,68}$ The results in Figure 4 indicate the palmitoyl chains have lower order than the stearic chains, and thus have a reduced projection along the $z$-axis. Coupled with the topological difference of two fewer methylene groups, our findings suggest the possibility for an acyl chain length mismatch in this system. To examine this mismatch quantitatively, we show in Figure 5 the average chain

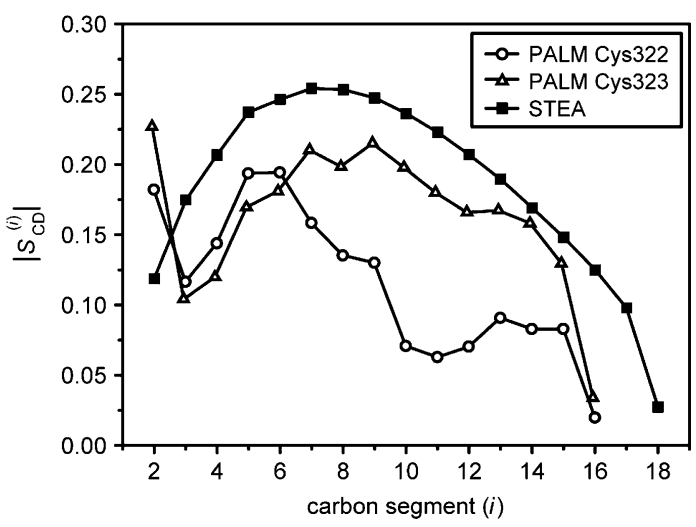

Figure 4. Calculated ${ }^{2} \mathrm{H}$ solid-state NMR order parameters for the lipid modifications of rhodopsin as well as the STEA chains of the surrounding lipids as a function of carbon position. The order parameters of the STEA chains ( $\mathbf{\square})$ were averaged over all lipids. Order parameters of the lipid modifications were evaluated individually for the modification attached to Cys322 (O) and Cys323 $(\triangle)$. Note that the order parameters of the palmitoylation at Cys322 are very low in comparison to the surrounding saturated chains of the lipids. The order parameters of the palmitoylation at Cys323 fall between these two extremes.

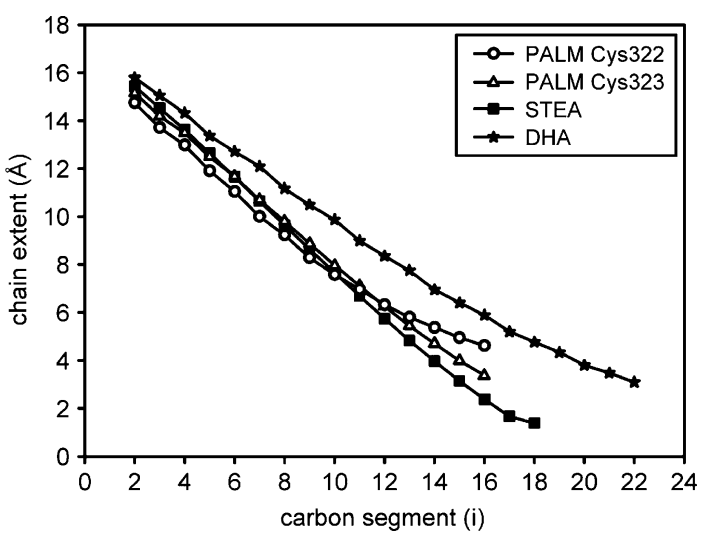

Figure 5. Plot of the average position of each carbon along the membrane normal with respect to the membrane center whose position was fixed at zero. Data are shown separately for the DHA $(\star)$ and STEA $(\boldsymbol{\square})$ chains as well as for lipid modifications attached to Cys322 (O) and Cys323 $(\triangle)$. Both lipid modifications originate close to the top of the lipid acyl chains. However their terminal $\mathrm{CH}_{3}$ groups are on average located at considerable distances from the membrane center yielding a substantial mismatch in chain length.

extent for each of the lipid modifications, as well as for the stearoyl and the DHA chain of the phospholipids. In this plot, the average distance of each carbon is shown from the membrane center, whose position is defined to be located at $z=$ 0 (alternatively chain extension plots can be calculated by defining the terminal $\mathrm{CH}_{3}$ group at $z=0$, but would lack the information of the distance from the actual membrane center $\left.^{60,68}\right)$. As anticipated, the lower order parameters combined with the smaller number of methylene groups produces a hydrophobic monolayer thickness mismatch of $\sim 3$ A. Specifically, Figure 5 shows that the palmitoyl and stearoyl carbonyl groups are approximately coplanar, but that the chain methyl groups are located on average in distinctly different regions of the bilayer. This finding is in striking opposition to the observations of lipid modifications in the peripheral membrane protein N-ras, where the post-translational mod- 
ifications have been shown to precisely adjust to the thickness of the lipid bilayer matrix. ${ }^{69}$

\section{DISCUSSION}

Overall we discovered that the two post-translational palmitoyl modifications of rhodopsin exhibit a rather unexpected behavior in a nativelike membrane environment. Regarding their contacts with the surrounding lipids, the palmitoyl groups clearly prefer the highly unsaturated chains compared to saturated lipid acyl chains. Both palmitoylations are characterized by very large fluctuations of their conformations, leading to very low order parameters, and a relatively short average penetration depth in the membrane. Interestingly, they often make contact with the transmembrane helices of rhodopsin; this mostly involves helix $\mathrm{H} 1$ between residues 48-61 with Pro53 showing the largest number of contacts followed by Phe56. When the palmitoyl groups are in contact with the protein they frequently exhibit very distorted and kinked conformations, leading to the observed low order parameters. Most striking, however, are the differences observed between the two palmitoylations despite their close proximity as thioesters of the two neighboring cysteines. The palmitoylation at Cys 322 consistently makes contacts with the transmembrane helices, while the palmitoylation at Cys 323 makes many more contacts with the surrounding lipids. The fluctuations and contacts of the palmitoylations are summarized in Figure 6, where the excursions of the palmitoyl chains of Cys 322 and Cys323 are depicted via projection of their terminal methyl group density onto the plane of the bilayer. A cluster of high
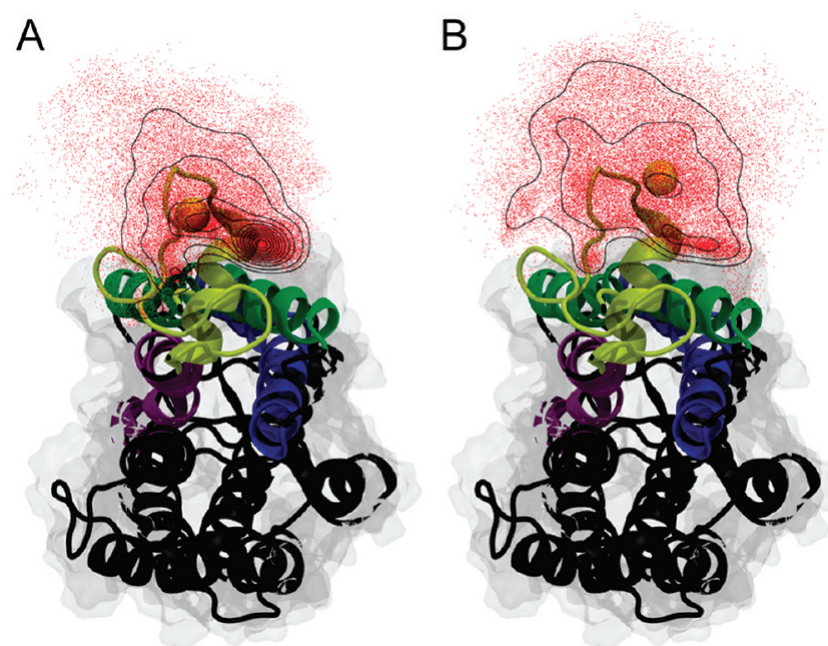

Figure 6. View of rhodopsin looking along the membrane normal from the cytoplasmic side (corresponding to the cell interior) toward the extracellular side. The relative positions of the terminal methyl groups of the palmitoyl substituents attached to Cys322 (A) and Cys323 (B) were determined in 1-ns intervals. Their distribution is shown as red dots with black contour lines indicating their density. For reference purposes in both panels the average position of the cysteine residue to which the lipid modification is attached is shown as a yellow vdW sphere. Helices H1 (green), H2 (blue), H7 (purple), and H8 (yellow) are color-coded with the remaining helices shown in black. The palmitoylation at Cys322 shows a clear accumulation at the protein/lipid interface. In contrast the palmitoylation at Cys323 is more evenly distributed about the cysteine residue and only shows minor accumulation close to helix $\mathrm{H} 1$. Rhodopsin was rendered using VMD. ${ }^{67}$ density is evident near helix $\mathrm{H} 1$ for Cys322 (Figure 6A), while the terminal methyl group on Cys323 (Figure 6B) is more evenly distributed. This arrangement reflects previous findings (Figure 2) for the close contacts of the palmitoylations to helix H1.

The observed differences between the two palmitoylations are surprising considering previous studies of proteins with multiple palmitoylations, which showed them to be virtually indistinguishable. ${ }^{52,54}$ However, closer inspection of the average position of the thioester sulfurs (indicated as yellow spheres in Figure 6) indicates the palmitoylation at Cys323 is on average farther removed from the protein than at Cys322. This finding suggests that Cys323 pays a larger entropic penalty for association with the protein compared to the palmitoylation at Cys322. Our interpretation is consistent with previous work that explains the preferential solvation of rhodopsin by highly unsaturated DHA chains. ${ }^{35}$ Using a rotational isomeric model of lipid acyl chain conformations, it was shown that acyl chains are repelled from the protein surface due to a loss of chain entropy upon association and that this entropic penalty is much smaller for unsaturated chains. ${ }^{70}$ Because the saturated palmitoylations are covalently attached to the protein, they do not have the opportunity to avail themselves of the favorable chain entropy associated with leaving the immediate protein environment. Thus the saturated palmitoyl chains covalently bound to the protein can behave quite differently from saturated chains attached to phospholipids with greater translational and configurational freedom. This discussion also concerns the relevance of our findings for other GPCRs. Rhodopsin is a special case among GPCRs as it is embedded in membranes with very high levels of unsaturation atypical of average cell membranes and is also present at very high concentrations compared to other GPCRs. However, we contend that lower GPCR concentrations or more saturated lipids would increase the tendency of the palmitoylations to associate with the protein. Lower GPCR concentrations would increase the entropic penalty of the phospholipids to associate with the protein; and increasing the saturation level of the membrane would reduce the number of lipids that preferentially associate with the protein; thus both effects would work to increase contact between the protein and palmitoylations. This suggests that our findings are likely relevant for other GPCRs.

Most striking, the unique distributions of the two chains suggest multiple roles for the palmitoyl modifications. The graph of chain extent along the bilayer (Figure 5) is particularly illuminating of this point. It shows that the upper chain segments of the palmitoylations are approximately coplanar with their phospholipid analogs, but that the extent of acyl chain alignment diminishes as the methyl termini are approached. This behavior indicates the palmitoyl chains undergo more extensive fluctuations compared to the stearoyl chains at the expense of tighter chain packing. An additional effect is that the palmitoylations do not reach to the center of the bilayer, which is energetically unfavorable. This conclusion follows both because of the absence of interactions with the last few STEA methylene segments, and because it requires paying an energy penalty to place the bulky palmitoyl methyl groups away from the bilayer center, where the free volume is maximized. Such a misalignment could influence the balance of curvature stress and hydrophobic matching within the bilayer that affects the equilibrium between the MI and MII substates 
in rhodopsin activation, in accord with the flexible surface model. ${ }^{32}$

It is well accepted that post-translational lipid modifications can serve very diverse functions for peripheral membrane proteins. For example, a kinetic membrane trapping model has been proposed such that a protein transiently binds to different membranes until it arrives at the correct subcellular location, where it becomes palmitoylated and therefore permanently anchored. ${ }^{71}$ It was also shown that palmitoylated peripheral membrane proteins preferentially localize at domain boundaries. ${ }^{69}$ For myristoylated peripheral membrane proteins a switch mechanism is described, where the myristoylation is buried in the protein such that the protein is soluble and inactive. Upon activation the lipid modification becomes exposed and leads to binding of the protein to the membrane. ${ }^{72}$ In GPCRs the case against palmitoyl groups solely as membrane anchors is strengthened by recent NMR and MD studies of the properties of post-translational lipid modifications of peripheral membrane proteins, where the anchoring role of the acylations is well established. $53,54,69,73$ These studies show that the lipid modifications adjust their structures to accommodate the thickness of the membrane bilayer, even when the hydrophobic thickness was increased by nearly $100 \% .{ }^{69}$ Additionally, no evidence was seen in these systems for contact between the acyl chains and the proteins; instead the acyl chains maximized their contact with phospholipids.

Multiple roles for post-translational lipid modifications are also supported by an examination of GPCR crystal structures in the protein data bank. Since the publication of the first crystal structure of rhodopsin in $2000,{ }^{3}$ several additional structures have been determined. A number of these structures have resolved both lipid modifications, ${ }^{42-51}$ suggesting that the structural disorder observed in the simulations is not present in the environment of a crystal, even with the presence of detergents. Nevertheless, it is worthwhile to analyze the distribution of conformations to establish a connection to our results. Although a diverse set of conformations is observed, it is interesting that the greatest number show only the lipid modification attached to Cys322 in contact with the helix bundle. $^{43,46,48,50,51,58}$ While a few structures show both modifications in close contact with the transmembrane helices, ${ }^{45,47,49}$ none of the entries in the database show the Cys323 palmitoylation contacting the protein while Cys322 does not. Additionally, a few of the structures show the lipid modifications removed from the parent helix bundle, and most likely in contact with neighboring proteins in the crystal. ${ }^{42,44}$ The close contacts observed between the palmitoylations and the helical bundle in the GPCR X-ray structures could easily be seen as artifacts of the crystal packing. Yet the observation of repeated contacts observed over more than a microsecond of temporal sampling, and across 26 unique lipid bilayer arrangements suggests this motif may play a yet unidentified functional role in this protein class.

Although these simulations do not in themselves suggest what this role might be, our results cast doubt on the notion that the role of the palmitoylations entails mainly acting as nonspecific anchors. This assertion is based on both the frequent contacts with the protein that necessarily reduce interactions with the lipid bilayer chains, and on the details of the palmitoyl conformations observed in the simulation. For example, the more diffuse distribution of Cys323 implies a potential role as a lipid anchor. Nevertheless, it is tempting to speculate that it might act more specifically as it clearly prefers unsaturated over saturated chains, and can therefore influence the orientation, subcellular location, or diffusion properties of rhodopsin. In addition, it could also be available in the membrane to make contact with other proteins mediating interactions with rhodopsin. By contrast, the palmitoyl-protein interaction pattern observed for Cys322 suggests a specific interaction with the transmembrane helices. This interaction might stabilize, or potentially destabilize, the dark state of rhodopsin or modulate the lipid-protein interactions to which rhodopsin reacts very sensitively. However, it might also interfere with any of the substates that rhodopsin adopts during activation.

Our analysis, based on molecular simulations of rhodopsin, suggests multiple possibilities for experimental studies to examine this and related systems. For example, experimental validation of predicted NMR observables would address our contention that the acylations in rhodopsin differ in significant ways from those of peripheral membrane proteins. Furthermore, given our observations that the two palmitoylations attached to neighboring cysteines of rhodopsin behave very differently, it would be interesting to examine the structure and function of pairs of lipid modifications of other GPCRs.

\section{ASSOCIATED CONTENT}

\section{Supporting Information}

Radial distribution functions of the palmitoylations with lipids comprising a PC or PE headgroup; correlation functions of the $\mathrm{C}-\mathrm{H}$ bond vectors of carbons $\mathrm{C} 3, \mathrm{C} 9$, and $\mathrm{C} 15$ of both palmitoylations; and complete ref 61 . This material is available free of charge via the Internet at http://pubs.acs.org.

\section{AUTHOR INFORMATION}

\section{Corresponding Author}

alexander.vogel@medizin.uni-leipzig.de

Notes

The authors declare no competing financial interest.

\section{ACKNOWLEDGMENTS}

Financial support from the U.S. National Science Foundation (MCB-0950258 to S.E.F.), the U.S. National Institutes of Health (GM095496 to A.G. and EY012049 and EY018891 to M.F.B.) and the "Exzellenznetzwerk Biowissenschaften" funded by the federal state of Sachsen-Anhalt is gratefully acknowledged. B.E.S.O. thanks Milton T. Stubbs and Andrea Sinz for financial support.

\section{REFERENCES}

(1) Schoneberg, T.; Schultz, G.; Gudermann, T. Mol. Cell. Endocrinol. 1999, 151, 181-193.

(2) Gloriam, D. E.; Foord, S. M.; Blaney, F. E.; Garland, S. L. J. Med. Chem. 2009, 52, 4429-4442.

(3) Palczewski, K.; Kumasaka, T.; Hori, T.; Behnke, C. A.; Motoshima, H.; Fox, B. A.; Le Trong, I.; Teller, D. C.; Okada, T.; Stenkamp, R. E.; Yamamoto, M.; Miyano, M. Science 2000, 289, 739745 .

(4) Altenbach, C.; Kusnetzow, A. K.; Ernst, O. P.; Hofmann, K. P.; Hubbell, W. L. Proc. Natl. Acad. Sci. U.S.A. 2008, 105, 7439-7444.

(5) Ahuja, S.; Crocker, E.; Eilers, M.; Hornak, V.; Hirshfeld, A.; Ziliox, M.; Syrett, N.; Reeves, P. J.; Khorana, H. G.; Sheves, M.; Smith, S. O. J. Biol. Chem. 2009, 284, 10190-10201.

(6) Salgado, G. F.; Struts, A. V.; Tanaka, K.; Krane, S.; Nakanishi, K.; Brown, M. F. J. Am. Chem. Soc. 2006, 128, 11067-11071.

(7) Salgado, G. F. J.; Struts, A. V.; Tanaka, K.; Fujioka, N.; Nakanishi, K.; Brown, M. F. Biochemistry 2004, 43, 12819-12828. 
(8) Struts, A. V.; Salgado, G. F.; Brown, M. F. Proc. Natl. Acad. Sci. U.S.A. 2011, 108, 8263-8268.

(9) Swift, S.; Leger, A. J.; Talavera, J.; Lei, Z.; Bohm, A.; Kuliopulos, A. J. Biol. Chem. 2006, 281, 4109-4116.

(10) Chien, E. Y. T.; Liu, W.; Zhao, Q. A.; Katritch, V.; Han, G. W.; Hanson, M. A.; Shi, L.; Newman, A. H.; Javitch, J. A.; Cherezov, V.; Stevens, R. C. Science 2010, 330, 1091-1095.

(11) Rasmussen, S. G. F.; Choi, H. J.; Rosenbaum, D. M.; Kobilka, T. S.; Thian, F. S.; Edwards, P. C.; Burghammer, M.; Ratnala, V. R. P.; Sanishvili, R.; Fischetti, R. F.; Schertler, G. F. X.; Weis, W. I.; Kobilka, B. K. Nature 2007, 450, 383-387.

(12) Warne, T.; Serrano-Vega, M. J.; Baker, J. G.; Moukhametzianov, R.; Edwards, P. C.; Henderson, R.; Leslie, A. G. W.; Tate, C. G.; Schertler, G. F. X. Nature 2008, 454, 486-491.

(13) Jaakola, V. P.; Griffith, M. T.; Hanson, M. A.; Cherezov, V.; Chien, E. Y. T.; Lane, J. R.; IJzerman, A. P.; Stevens, R. C. Science 2008, 322, 1211-1217.

(14) Shimamura, T.; Shiroishi, M.; Weyand, S.; Tsujimoto, H.; Winter, G.; Katritch, V.; Abagyan, R.; Cherezov, V.; Liu, W.; Han, G. W.; Kobayashi, T.; Stevens, R. C.; Iwata, S. Nature 2011, 475, 65U82.

(15) Delos Santos, N. M.; Gardner, L. A.; White, S. W.; Bahouth, S. W. J. Biol. Chem. 2006, 281, 12896-12907.

(16) Feng, G. J.; Kellett, E.; Scorer, C. A.; Wilde, J.; White, J. H.; Milligan, G. J. Biol. Chem. 2003, 278, 33400-33407.

(17) Phillips, W. J.; Cerione, R. A. J. Biol. Chem. 1992, 267, 1703217039.

(18) Fritze, O.; Filipek, S.; Kuksa, V.; Palczewski, K.; Hofmann, K. P.; Ernst, O. P. Proc. Natl. Acad. Sci. U.S.A. 2003, 100, 2290-2295.

(19) Ernst, O. P.; Meyer, C. K.; Marin, E. P.; Henklein, P.; Fu, W. Y.; Sakmar, T. P.; Hofmann, K. P. J. Biol. Chem. 2000, 275, 1937-1943.

(20) Resh, M. D. Methods 2006, 40, 191-197.

(21) Miggin, S. M.; Lawler, O. A.; Kinsella, B. T. J. Biol. Chem. 2003, 278, 6947-6958.

(22) O’Dowd, B. F.; Hnatowich, M.; Caron, M. G.; Lefkowitz, R. J.; Bouvier, M. J. Biol. Chem. 1989, 264, 7564-7569.

(23) Reid, H. M.; Kinsella, B. T. Cellular Signalling 2007, 19, 10561070.

(24) Reid, H. M.; Mulvaney, E. P.; Turner, E. C.; Kinsella, B. T. J. Biol. Chem. 2010, 285, 18709-18726.

(25) Gehret, A. U.; Jones, B. W.; Tran, P. N.; Cook, L. B.; Greuber, E. K.; Hinkle, P. M. Mol. Pharmacol. 2010, 77, 288-297.

(26) Blanpain, C.; Wittamer, V.; Vanderwinden, J. M.; Boom, A.; Renneboog, B.; Lee, B.; Le Poul, E.; El Asmar, L.; Govaerts, C.; Vassart, G.; Doms, R. W.; Parmentier, M. J. Biol. Chem. 2001, 276, 23795-23804.

(27) Karnik, S. S.; Ridge, K. D.; Bhattacharya, S.; Khorana, H. G. Proc. Natl. Acad. Sci. U.S.A. 1993, 90, 40-44.

(28) Morozova, D.; Weiss, M. Biophys. J. 2010, 98, 800-804.

(29) Ovchinnikov, Y. A.; Abdulaev, N. G.; Bogachuk, A. S. FEBS Lett.

1988, 230, 1-5.

(30) Park, P. S. H.; Sapra, K. T.; Jastrzebska, B.; Maeda, T.; Maeda, A.; Pulawski, W.; Kono, M.; Lem, J.; Crouch, R. K.; Filipek, S.; Muller,

D. J.; Palczewski, K. Biochemistry 2009, 48, 4294-4304.

(31) Brown, M. F. Curr. Top. Membr. 1997, 44, 285-356.

(32) Botelho, A. V.; Gibson, N. J.; Thurmond, R. L.; Wang, Y.; Brown, M. F. Biochemistry 2002, 41, 6354-6368.

(33) Soubias, O.; Teague, W. E.; Hines, K. G.; Mitchell, D. C.; Gawrisch, K. Biophys. J. 2010, 99, 817-824.

(34) Zaitseva, E.; Brown, M. F.; Vogel, R. J. Am. Chem. Soc. 2010, 132, 4815-4821.

(35) Grossfield, A.; Feller, S. E.; Pitman, M. C. Proc. Natl. Acad. Sci. U.S.A. 2006, 103, 4888-4893.

(36) Feller, S. E.; Gawrisch, K.; Woolf, T. B. J. Am. Chem. Soc. 2003, $125,4434-4435$.

(37) Rajamoorthi, K.; Petrache, H. I.; McIntosh, T. J.; Brown, M. F. J. Am. Chem. Soc. 2005, 127, 1576-1588.

(38) Petrache, H. I.; Salmon, A.; Brown, M. F. J. Am. Chem. Soc. 2001, 123, 12611-12622.
(39) Feller, S. E.; Gawrisch, K.; MacKerell, A. D. J. Am. Chem. Soc. 2002, 124, 318-326.

(40) Wassall, S. R.; Brzustowicz, M. R.; Shaikh, S. R.; Cherezov, V.; Caffrey, M.; Stillwell, W. Chem. Phys. Lipids 2004, 132, 79-88.

(41) Moench, S. J.; Moreland, J.; Stewart, D. H.; Dewey, T. G. Biochemistry 1994, 33, 5791-5796.

(42) Standfuss, J.; Edwards, P. C.; D’Antona, A.; Fransen, M.; Xie, G.; Oprian, D. D.; Schertler, G. F., X Nature 2011, 471, 656-660.

(43) Makino, C. L.; Riley, C. K.; Looney, J.; Crouch, R. K.; Okada, T. Biophys. J. 2010, 99, 2366-2373.

(44) Scheerer, P.; Park, J. H.; Hildebrand, P. W.; Kim, Y. J.; Krauss, N.; Choe, H. W.; Hofmann, K. P.; Ernst, O. P. Nature 2008, 455, 497-U30.

(45) Shimamura, T.; Hiraki, K.; Takahashi, N.; Hori, T.; Ago, H.; Masuda, K.; Takio, K.; Ishiguro, M.; Miyano, M. J. Biol. Chem. 2008, 283, 17753-17756.

(46) Nakamichi, H.; Buss, V.; Okada, T. Biophys. J. 2007, 92, L106L108.

(47) Salom, D.; Lodowski, D. T.; Stenkamp, R. E.; Le Trong, I.; Golczak, M.; Jastrzebska, B.; Harris, T.; Ballesteros, J. A.; Palczewski, K. Proc. Natl. Acad. Sci. U.S.A. 2006, 103, 16123-16128.

(48) Nakamichi, H.; Okada, T. Proc. Natl. Acad. Sci. U.S.A. 2006, 103, 12729-12734.

(49) Li, J.; Edwards, P. C.; Burghammer, M.; Villa, C.; Schertler, G. F. X. J. Mol. Biol. 2004, 343, 1409-1438.

(50) Okada, T.; Fujiyoshi, Y.; Silow, M.; Navarro, J.; Landau, E. M.; Shichida, Y. Proc. Natl. Acad. Sci. U.S.A. 2002, 99, 5982-5987.

(51) Teller, D. C.; Okada, T.; Behnke, C. A.; Palczewski, K.; Stenkamp, R. E. Biochemistry 2001, 40, 7761-7772.

(52) Huster, D.; Vogel, A.; Katzka, C.; Scheidt, H. A.; Binder, H.; Dante, S.; Gutberlet, T.; Zschörnig, O.; Waldmann, H.; Arnold, K. J. Am. Chem. Soc. 2003, 125, 4070-4079.

(53) Vogel, A.; Katzka, C. P.; Waldmann, H.; Arnold, K.; Brown, M. F.; Huster, D. J. Am. Chem. Soc. 2005, 127, 12263-12272.

(54) Vogel, A.; Tan, K. T.; Waldmann, H.; Feller, S. E.; Brown, M. F.; Huster, D. Biophys. J. 2007, 93, 2697-2712.

(55) Dror, R. O.; Arlow, D. H.; Borhani, D. W.; Jensen, M. O.; Piana, S.; Shaw, D. E. Proc. Natl. Acad. Sci. U.S.A. 2009, 106, 4689-4694.

(56) Roy, J.; Laughton, C. A. Biophys. J. 2010, 99, 218-226.

(57) Lau, P. W.; Grossfield, A.; Feller, S. E.; Pitman, M. C.; Brown, M. F. J. Mol. Biol. 2007, 372, 906-917.

(58) Okada, T.; Sugihara, M.; Bondar, A. N.; Elstner, M.; Entel, P.; Buss, V. J. Mol. Biol. 2004, 342, 571-583.

(59) Berman, H. M.; Westbrook, J.; Feng, Z.; Gilliland, G.; Bhat, T. N.; Weissig, H.; Shindyalov, I. N.; Bourne, P. E. Nucleic Acids Res. 2000, 28, 235-242.

(60) Petrache, H. I.; Dodd, S. W.; Brown, M. F. Biophys. J. 2000, 79, 3172-3192.

(61) MacKerell, A. D.; et al. J. Phys. Chem. B 1998, 102, 3586-3616.

(62) Klauda, J. B.; Brooks, B. R.; MacKerell, A. D.; Venable, R. M.; Pastor, R. W. J. Phys. Chem. B 2005, 109, 5300-5311.

(63) Pitman, M. C.; Suits, F.; MacKerell, A. D.; Feller, S. E. Biochemistry 2004, 43, 15318-15328.

(64) Hockney, R. W.; Eastwood, J. W. Computer simulation using particles; McGraw-Hill: New York, 1981.

(65) Pollock, E. L.; Glosli, J. Comput. Phys. Commun. 1996, 95, 93110.

(66) Andersen, H. C. J. Comput. Phys. 1983, 52, 24-34.

(67) Humphrey, W.; Dalke, A.; Schulten, K. J. Mol. Graphics 1996, $14,33-\&$.

(68) Petrache, H. I.; Tu, K. C.; Nagle, J. F. Biophys. J. 1999, 76, 2479-2487.

(69) Vogel, A.; Reuther, G.; Weise, K.; Triola, G.; Nikolaus, J.; Tan, K. T.; Nowak, C.; Herrmann, A.; Waldmann, H.; Winter, R.; Huster, D. Angew. Chem., Int. Ed. 2009, 48, 8784-8787.

(70) Grossfield, A.; Feller, S. E.; Pitman, M. C. J. Phys. Chem. B 2006, $110,8907-8909$.

(71) Dunphy, J. T.; Linder, M. E. Biochim. Biophys. Acta 1998, 1436, 245-261. 
(72) Ames, J. B.; Ishima, R.; Tanaka, T.; Gordon, J. I.; Stryer, L.; Ikura, M. Nature 1997, 389, 198-202.

(73) Scheidt, H. A.; Huster, D. Biophys. J. 2009, 96, 3663-3672. 